\title{
High Prevalence and Risk Factors for Hookworm and Strongyloides Stercoralis Infections in Rural East Kalimantan, Indonesia
}

\author{
Jitbanjong Tangpong ( $\sim$ rjitbanj@wu.ac.th ) \\ Walailak University https://orcid.org/0000-0002-4768-7829 \\ Blego Sedionoto \\ Walailak University, School of Allied Health Sciences \\ Sueptrakool Wasessombat \\ Walailak University \\ Chuchard Punsawad \\ Walailak University \\ Witthaya Anamnart \\ Walailak University \\ Manas Kotepui \\ Walailak University
}

Research

Keywords: hookworm, S. stercoralis, risk factors, Kalimantan, Indonesia

Posted Date: January 27th, 2021

DOI: https://doi.org/10.21203/rs.3.rs-154049/v1

License: (1) This work is licensed under a Creative Commons Attribution 4.0 International License. Read Full License 


\section{Abstract}

Background: Hookworm and Strongyloides stercoralis infections are neglected tropical diseases in developing countries, which have environmental risk factors with the potential for transmission of hookworm and S. stercoralis infections. This study aimed to explore the prevalence and risk factors for hookworm and S. stercoralis infections in East Kalimantan Province, Indonesia.

Methods: A cross-sectional study enrolled 213 participants from 28 villages, including 12 villages from Muara Kaman District and 16 villages from Marangkayu District in East Kalimantan Province. The data from 10-15 households of each village with 1-3 participants in each household were collected. In this study, Kato Katz and Koga agar plate cultures/KAP cultures were used for diagnosing hookworm and $S$. stercoralis infections among participants. The Pearson chi-square analysis was used to analyze the correlation between risk factors with hookworm and S. stercoralis infections.

Results: In this study, 72 (33.8\%) and 17 (8.0\%) participants were infected with hookworm and $S$. stercoralis, respectively. The prevalence of hookworm infection reported was higher than $S$. stercoralis infection in rural areas of East Kalimantan Province. Hookworm infection has been significantly associated with demographic factors, sanitation conditions and personal hygiene. In addition, high hookworm infection has been significantly associated with environmental factors such as locality of district, organic carbon content in the soil, temperature, humidity, number of days of rainfall, volume of rainfall, wet/dry soil around houses, and habitation. However, S. stercoralis infection has not been significantly correlated with demographic factors, sanitation conditions, and personal hygiene. The highest odds ratio of hookworm infection was in village areas surrounded by a river or coastal areas OR: 2.92 (95\% CL: 1.38-6.24), while the highest odds ratio of $S$. stercoralis infection was in households with habitation with humans infected with S. stercoralis OR: 95\% CL: 8.33 (2.27-30.61).

Conclusions: The high prevalence and risk factors of hookworm and S. stercoralis infections in rural communities in East Kalimantan Province. Characteristics of demographic factors, sanitation conditions, and personal hygiene that were correlated with hookworm and S. stercoralis infection should be used for control and prevention programs to reduce hookworm and S. stercoralis infections, especially in rural areas.

\section{Background}

Hookworm and Strongyloides stercoralis are two soil-transmitted helminths (STH) that are highly prevalent infections and serious public health concerns globally. Hookworm causes the highest public health burden among STH, while S. stercoralis causes long-lasting infections and significant morbidity. These two parasites are highly prevalent in poor rural communities in tropical and subtropical areas in many developing countries (1). They are transmitted through unprotected contact with soil that is endemic in tropical and temperate regions. In 2010, the prevalence of hookworm infection and $S$. stercoralis infection was estimated in 438.9 million people and 100 million people, respectively. Almost 
$70 \%$ of these infections occurred in Asia (2-4). Humans acquire hookworm and S. stercoralis infections through direct skin contact with infective third-stage larvae, where soil that was contaminated by human feces penetrate the intact human skin and eventually reach the small intestine (5). Generally, hookworm and $S$. stercoralis infections are found among poor people with poor environmental sanitation and where the climate is warm and humid $(6,7)$.

Factors affecting the difference in the prevalence of hookworm infection and $S$. stercoralis infection may include good hygiene practices among the population, availability of a sewage system, and length of the rainy season. Environmental factors have contributed to the transmission of diseases as well as to the growth and development of the worms $(8,9)$. Environmental factors, especially the long rainy season, may affect the decrease in the prevalence of $S$. stercoralis infection but not hookworm infection. A previous study demonstrated that the prevalence of $S$. stercoralis infection in Southern Thailand is lower than in other parts of the country. However, the prevalence of hookworm infection is still high in Southern Thailand because of the 10-months-long rainy season in Southern Thailand in contrast with the 4months-long rainy season in other parts (10). Another study in Cambodia reported a lower prevalence of $S$. stercoralis infection in the areas with heavy rainfall than in low rainfall areas, where the high amount of the organic carbon content in the soil affects the lower prevalence of $S$. stercoralis infection (11). A previous study in Southern Laos demonstrated hookworm infection and $S$. stercoralis infection at about $56.1 \%$ and $41 \%$, respectively (12).

Kutai Kertanegara Regency, East Kalimantan Province, Indonesia is located at $0.44019^{\circ} \mathrm{S}$ and $116.98139^{\circ} \mathrm{E}$. The average temperature is $28^{\circ} \mathrm{C}\left(26-32^{\circ} \mathrm{C}\right)$. Muara Kaman District is located at the surrounding Mahakam River and is close in proximity with forest areas and palm plantations. In contrast, Marangkayu District is located in a coastal area and surrounded by rubber plantations, palm plantations, and rice fields. We collected data from 28 villages, consisting of 12 villages from Muara Kaman District and 16 villages from Marangkayu District. The prevalence of hookworm and S. stercoralis infections in rural East Kalimantan Province, Indonesia, remains unclear. Because the environment is suitable for hookworm and S. stercoralis infections in this area, it is important to determine the prevalence and risk factors for these two parasites. This study aimed to explore the prevalence and risk factors (including soil quality, surrounding vegetation, temperature, humidity, village areas, length of rainy season, type of pets (cats or dogs), personal hygiene, and sanitation conditions of households) for hookworm and $S$. stercoralis infections in East Kalimantan Province, Indonesia.

\section{Methods}

\section{Study design}

This cross-sectional study was conducted from July 2018 to September 2019 in rural areas of Muara Kaman District and Marangkayu District, Kutai Kertanegara Regency, East Kalimantan Province, Indonesia. Data was collected from 10-15 households in each village with 1-3 participants in each 
household. The demographic data was collected from 213 participants and was analyzed with the environmental factors (13).

\section{Study population, sample size, and sampling technique}

Two districts in Kutai Kertanegara Regency, East Kalimantan Province, were selected for the purpose of this study: Muara Kaman District and Marangkayu District (Fig. 1), where these areas have differences in soil quality, number of days and yearly volume of rainfall, temperature, humidity, elevation above sea level, village areas, and surrounding vegetation. These areas were selected based on the potential risk of hookworm and $S$. stercoralis infections due to the following characteristics: rural areas, poor sanitation and hygiene practices, agricultural activity, and surrounding forests. The sample size was calculated using a prevalence rate (p) of $55.4 \%$ as detailed from a previous study (23), with a $95 \%$ confidence interval $(z=1.96)$ and a $10 \%$ margin of error $(d=0.1)$. Therefore, the calculated sample size was 95 participants per district. However, the final sample size would end up being reduced by around $15 \%$ due to subjects being unable to pass stool on the study date. Thus, the aim was for a sample size of 110 participants per district. A simple random sampling method was used to select the population from each district. Inclusion criteria for participants included the following: $\geq 2$ years old, lived in both areas, and were able to complete questionnaires and provide stool samples. One hundred and ten participants per area were randomly selected and were given the instructions, and they were also provided the questionnaires and plastic containers for stool sample collection. In total, 220 participants were enrolled and completed the questionnaires. Seven participants were excluded from the study because they did not send in their stool samples. In total, 213 participants were included for this study.

\section{Data collection and laboratory processing}

The stool samples were collected and sent to The Biomedical Laboratory, School of Public Health, Mulawarman University. Two methods were performed to identify the parasites including agar plate culture and the Kato-Katz technique. A modified agar plate culture was performed as described by Koga et al., 1991. Briefly, approximately 2 grams of stool sample was placed at the center of nutrient agar and kept at room temperature for five days. Tracks from larva crawling and larvae or adult worms were observed under an inversed microscope. If any tract was spotted, $10 \mathrm{ml}$ of $10 \%$ formalin was added to the agar surface for 5-10 minutes and transferred to a centrifuged tube. Centrifugation of suspension was performed at 2,500 rpm for 5 minutes, and then the supernatant was discarded. The sediment was further examined for hookworm and S. stercoralis larvae or adult worms. For the Kato-Katz thick smear, $50 \mathrm{mg}$ of stool was placed on a slide and covered with a cellophane paper soaked in glycerin solution for 24 hours. The stool was spread out using a rubber stick and examined after 30 minutes, and the eggs of parasites were counted (14-16). The environmental conditions of houses surrounding the village were recorded on the same day or following the day after stool samples were collected.

\section{Data analysis}


Demographic data and personal hygiene of the participants were collected by questionnaires. The sanitation conditions of each household of the participants were collected by observation. Environmental data including surrounding vegetation, elevation of soil above sea level, type of soil around houses, type of pets, length of the rainy season, humidity, and temperature per year were collected. The quality of soil including the organic carbon content, clay content, and $\mathrm{pH}$ was determined at the soil laboratory at Mulawarman University. The vegetation and type of soil around houses were collected by a standardizedobservation form. The types of pets were also recorded by researchers. The length of the rainy season, humidity, and temperature per year were collected from the Central Bureau of Statistics (https://www.bps.go.id) and the Central Bureau of Meteorology, Climatology, and Geophysical of Indonesia (https://www.bmkg.go.id). The prevalence of hookworm and S. stercoralis infections was stratified according to the demographic data, sanitation conditions and personal hygiene, and environmental data; and it was reported by descriptive statistic. Statistical analysis was performed by the Chi-square test using SPSS Version 22 (IBM SPSS Statistics for Windows, NY: IBM Corp). The association between the parasite infections and demographic data, sanitation conditions, personal hygiene, and environmental risk factors was analyzed using correlation analysis. The differences in demographic data, sanitation conditions, personal hygiene, and environmental risk factors were analyzed using the Chi-Square test with a confidence interval of $95 \%$. The level of significance for all tests was considered at $p<0.05$.

\section{Results}

\section{Demographic characteristics}

A total of 213 individuals from 28 villages participated in this study. Twelve villages were from Muara Kaman District and sixteen villages were from Marangkayu District, East Kalimantan Province, Indonesia (Fig. 1). Most of the participants (118/213,55.4\%) were from Marangkayu District, while 95 participants (44.6\%) were from Muara Kaman District. The age of all participants ranged from 2 years to 70 years old. Males $(130 / 213,61 \%)$ were the dominant participants. The main occupations of the participants were farmers $(70 / 213,32.9 \%)$, at home $(65 / 213,25.8 \%)$, at school $(81 / 213,38.0 \%)$, and other $(7 / 213,3.3 \%)$. The sanitation conditions of the participants included the type of floor covering at the door, type of yard covering surrounding the house, waste water treatment, water sources for daily activity and drinking, and toilets. Personal hygiene of the participants included wearing shoes in outdoor activities, washing feet and hands after soil contact, washing fruits or vegetables before eating, eating uncooked vegetables, washing hands after pet contact, and using toilets in the home (Table 1). 
Table 1

Characteristics of the 213 participants in the community-based study

\begin{tabular}{|c|c|c|}
\hline Variable & Category & $\mathrm{n}(\%)$ \\
\hline \multirow[t]{2}{*}{ Gender } & Male & $\begin{array}{l}130 \\
(61.0)\end{array}$ \\
\hline & Female & $83(39.0)$ \\
\hline \multirow[t]{2}{*}{ Age (years) } & $2-12$ & $114(53.5)$ \\
\hline & 13 and above & $99(46.5)$ \\
\hline \multirow[t]{4}{*}{ Main occupation } & Farmer & $70(32.9)$ \\
\hline & At home & $65(25.8)$ \\
\hline & At school & $81(38.0)$ \\
\hline & Other & $7(3.3)$ \\
\hline \multirow[t]{2}{*}{ Occupation } & Non-Agricultural & $75(64.8)$ \\
\hline & Agricultural & $\begin{array}{l}138 \\
(35.2)\end{array}$ \\
\hline \multirow[t]{2}{*}{ Type of indoor floor covering in the house } & Sanitary floor (cement, wood, etc.) & $\begin{array}{l}212 \\
(99.5)\end{array}$ \\
\hline & Soil floor & $1(0.5)$ \\
\hline \multirow[t]{2}{*}{ Type of yard covering } & Not soil & $35(16.4)$ \\
\hline & Soil & $\begin{array}{l}178 \\
(83.6)\end{array}$ \\
\hline \multirow[t]{2}{*}{ Waste water treatment } & Healthy waste water treatment & 108(50.7) \\
\hline & Without waste water treatment & $\begin{array}{l}105 \\
(49.3)\end{array}$ \\
\hline \multirow[t]{2}{*}{ Water sources for daily activity } & Sanitary water sources & $92(43.2)$ \\
\hline & Unsanitary water sources & $\begin{array}{l}121 \\
(56.8)\end{array}$ \\
\hline \multirow[t]{2}{*}{ Drinking water } & Sanitary drinking water & $117(54.9)$ \\
\hline & Unsanitary drinking water & $96(45.1)$ \\
\hline \multirow[t]{2}{*}{ Toilet } & Sanitary toilet in the home & $\begin{array}{l}181 \\
(85.0)\end{array}$ \\
\hline & $\begin{array}{l}\text { Open defecation (in plantation, } \\
\text { garden, or river) }\end{array}$ & $32(15.0)$ \\
\hline Using shoes outdoors & Routine & 74 (34.7) \\
\hline
\end{tabular}




\begin{tabular}{|c|c|c|}
\hline \multirow[t]{2}{*}{ Variable } & Category & $\mathrm{n}(\%)$ \\
\hline & Not routine & $\begin{array}{l}139 \\
(65.3)\end{array}$ \\
\hline \multirow[t]{2}{*}{ Washing feet after soil contact } & Yes & $26(12.2)$ \\
\hline & No & $\begin{array}{l}187 \\
(87.8)\end{array}$ \\
\hline \multirow[t]{2}{*}{ Washing fruits/vegetables before eating } & Routine & $33(15.5)$ \\
\hline & Not routine & $\begin{array}{l}180 \\
(84.5)\end{array}$ \\
\hline \multirow[t]{2}{*}{ Eating raw/uncooked fish/meat/vegetable } & No & $\begin{array}{l}172 \\
(80.8)\end{array}$ \\
\hline & Yes & $41(19.2)$ \\
\hline \multirow[t]{2}{*}{ Pet contact } & No & $\begin{array}{l}161 \\
(75.6)\end{array}$ \\
\hline & Yes & $52(24.4)$ \\
\hline \multirow[t]{2}{*}{ Washing hands after pet contact } & Yes & $4(1.9)$ \\
\hline & No & $\begin{array}{l}209 \\
(98.1)\end{array}$ \\
\hline \multirow[t]{2}{*}{ Washing hands after soil contact } & Yes & $25(11.7)$ \\
\hline & No & 188(88.3) \\
\hline \multirow[t]{2}{*}{ Washing feet before entering the house } & No & $13(6.1)$ \\
\hline & Yes & $\begin{array}{l}200 \\
(93.9)\end{array}$ \\
\hline \multirow[t]{2}{*}{ Using the toilet at home } & Yes & $\begin{array}{l}116 \\
(54.5)\end{array}$ \\
\hline & No & $97(45.5)$ \\
\hline \multirow[t]{2}{*}{ Using sandals at the toilet } & Routine & $\begin{array}{l}116 \\
(54.5)\end{array}$ \\
\hline & Not routine & $97(45.5)$ \\
\hline \multirow[t]{2}{*}{ District } & Muara Kaman & $95(44.6)$ \\
\hline & Marangkayu & $\begin{array}{l}118 \\
(55.4)\end{array}$ \\
\hline \multirow[t]{2}{*}{ Organic carbon content in soil (\%) } & $1.37-2.47$ & $91(42.7)$ \\
\hline & $>2.47-4.04$ & $122(57.3)$ \\
\hline
\end{tabular}




\begin{tabular}{|c|c|c|}
\hline Variable & Category & $\mathrm{n}(\%)$ \\
\hline \multirow[t]{2}{*}{$\mathrm{pH}$ of soil } & $4.26-5.85$ & $\begin{array}{l}102 \\
(47.9)\end{array}$ \\
\hline & $>5.85-6.92$ & $\begin{array}{l}111 \\
(52.1)\end{array}$ \\
\hline \multirow[t]{2}{*}{ Clay content in soil (\%) } & $4-18.5$ & $96(45.1)$ \\
\hline & $>18.5-42.50$ & $\begin{array}{l}117 \\
(54.9)\end{array}$ \\
\hline \multirow[t]{2}{*}{ Temperature $\left({ }^{0} \mathrm{C}\right)$} & $28-28.6$ & $\begin{array}{l}118 \\
(55.4)\end{array}$ \\
\hline & $>28.6-29.5$ & $95(44.6)$ \\
\hline \multirow[t]{2}{*}{ Humidity (\%) } & 65 & $\begin{array}{l}118 \\
(55.4)\end{array}$ \\
\hline & 66 & $95(44.6)$ \\
\hline \multirow[t]{2}{*}{ Number of days of rainfall (day) } & 164 & $95(44.6)$ \\
\hline & 174 & $\begin{array}{l}118 \\
(55.4)\end{array}$ \\
\hline \multirow[t]{2}{*}{ Rainfall volume $\left(\mathrm{mm}^{3}\right)$} & 3,549 & $95(44.6)$ \\
\hline & 4,000 & $\begin{array}{l}118 \\
(55.4)\end{array}$ \\
\hline \multirow[t]{2}{*}{ Elevation above sea level (m) } & $15-41.6$ & $97(45.5)$ \\
\hline & $>41.6-50$ & $\begin{array}{l}116 \\
(54.5)\end{array}$ \\
\hline \multirow[t]{2}{*}{ Texture of soil } & Sandy soil with organic material & $\begin{array}{l}139 \\
(65.3)\end{array}$ \\
\hline & $\begin{array}{l}\text { Non-sandy soil with organic } \\
\text { material }\end{array}$ & 74(37.3) \\
\hline \multirow[t]{2}{*}{ Vegetation } & $\begin{array}{l}\text { Surrounding palm plantations } \\
\text { and/or rubber plantations }\end{array}$ & $\begin{array}{l}167 \\
(78.4)\end{array}$ \\
\hline & Surrounding rice fields & $46(21.6)$ \\
\hline \multirow[t]{2}{*}{ Village areas } & Buffer river/sea & 166(77.9) \\
\hline & Hill area & $47(22.1)$ \\
\hline \multirow[t]{2}{*}{ Dry or wet soil surrounding the house } & Dry soil & 169(79.3) \\
\hline & Wet soil & $44(20.7)$ \\
\hline Having cats as pets & No & $80(37.6)$ \\
\hline
\end{tabular}




\begin{tabular}{|c|c|c|}
\hline Variable & Category & $\mathrm{n}(\%)$ \\
\hline & Yes & $\begin{array}{l}133 \\
(62.4)\end{array}$ \\
\hline \multirow[t]{2}{*}{ Having dogs as pets } & No & $4(1.9)$ \\
\hline & Yes & $\begin{array}{l}209 \\
(98.1)\end{array}$ \\
\hline \multirow[t]{2}{*}{ Hookworm in cats } & Negative & $80(37.6)$ \\
\hline & Positive & $\begin{array}{l}133 \\
(62.4)\end{array}$ \\
\hline \multirow[t]{2}{*}{ Hookworm in dogs } & Negative & $4(1.9)$ \\
\hline & Positive & $\begin{array}{l}209 \\
(98.1)\end{array}$ \\
\hline \multirow[t]{2}{*}{ S. stercoralis in cats } & Negative & $\begin{array}{l}134 \\
(62.9)\end{array}$ \\
\hline & Positive & $79(37.1)$ \\
\hline \multirow[t]{2}{*}{ S. stercoralis in dogs } & Negative & $52(24.4)$ \\
\hline & Positive & $\begin{array}{l}161 \\
(75.6)\end{array}$ \\
\hline \multirow[t]{2}{*}{$\begin{array}{l}\text { Habitation with humans infected with } S \text {. } \\
\text { stercoralis }\end{array}$} & No & $\begin{array}{l}194 \\
(91.1)\end{array}$ \\
\hline & Yes & $19(8.9)$ \\
\hline \multirow[t]{2}{*}{ Habitation with humans infected with hookworm } & No & $\begin{array}{l}152 \\
(71.4)\end{array}$ \\
\hline & Yes & $61(28.6)$ \\
\hline \multirow[t]{2}{*}{$\begin{array}{l}\text { Habitation with humans infected with hookworm } \\
\text { and S. stercoralis co-infection }\end{array}$} & No & $\begin{array}{l}196 \\
(92.0)\end{array}$ \\
\hline & Yes & $17(8.0)$ \\
\hline
\end{tabular}

The environmental data included the locality of the district, village areas, vegetation surrounding houses, elevation above sea level, carbon content in the soil, clay content in the soil, texture of the soil, dry or wet soil surrounding houses, humidity, temperature, length of the rainy season (number of days of rainfall yearly), volume of rainfall yearly, and type of pets (dogs or cats) (Table 1).

\section{Prevalence of hookworm and S. stercoralis infections}


Hookworm and S. stercoralis infections were examined using the Kato Katz technique and the APC method (Fig. 2). The results showed that 72 (33.8\%) stool samples were positive with hookworm. The prevalence of hookworm infection in Muara Kaman District $(53,55.8 \%)$ was higher than in Marangkayu District $(19,16.1 \%)$, while the prevalence of $S$. stercoralis infection in Marangkayu District $(14,11.9 \%)$ was higher than in Muara Kaman District (3, 3.2\%) (Fig. 3). The other details are listed in Table 2.

Table 2

Prevalence of hookworm and S. stercoralis infections in East Kalimantan Province

\begin{tabular}{|c|c|c|c|c|c|c|}
\hline \multirow[t]{2}{*}{ Infection } & \multicolumn{2}{|c|}{ Muara Kaman District } & \multicolumn{2}{|c|}{ Marangkayu District } & \multicolumn{2}{|l|}{ Total } \\
\hline & Positive & Negative & Positive & Negative & Positive & Negative \\
\hline Hookworm & $53(55.8 \%)$ & $42(44.2 \%)$ & $19(16.1 \%)$ & $99(83.9 \%)$ & $72(33.8 \%)$ & $141(66.2 \%)$ \\
\hline S. stercoralis & $3(3.2 \%)$ & $92(96.8 \%)$ & $14(11.9 \%)$ & $104(88.1 \%)$ & $17(8.0 \%)$ & $196(92 \%)$ \\
\hline Co-infection & $3(3.2 \%)$ & $92(96.8 \%)$ & $11(9.3 \%)$ & 107 (90.7\%) & $14(6.6 \%)$ & 199 (93.4\%) \\
\hline
\end{tabular}

\section{Risk factors of hookworm and S. stercoralis infections in East Kalimantan Province}

Participant characteristics and household sanitation conditions in relation to the prevalence of hookworm/S. stercoralis infections were determined. The results showed that hookworm infections were significantly correlated with age $(p=0.023)$, occupation $(p=0.044)$, water sources for daily activity $(p=$ $0.000)$, sources of drinking water $(p=0.000)$, and availability of toilets $(p=0.012)$ (Table 3 ). 
Table 3

The association between participant characteristics and the prevalence of hookworm/S. stercoralis infections

\begin{tabular}{|c|c|c|c|c|c|}
\hline \multirow[t]{2}{*}{ Variable } & \multirow[t]{2}{*}{ Category } & \multicolumn{2}{|c|}{ Positive n (\%) } & \multicolumn{2}{|l|}{$P$-value } \\
\hline & & Hookworm & $\begin{array}{l}\text { S. } \\
\text { stercoralis }\end{array}$ & Hookworm & $\begin{array}{l}\text { S. } \\
\text { stercoralis }\end{array}$ \\
\hline \multirow[t]{2}{*}{ Gender } & Male & $47(36.2)$ & $3(3.2)$ & \multirow[t]{2}{*}{0.364} & \multirow[t]{2}{*}{0.218} \\
\hline & Female & $25(30.1)$ & 14 (11.9) & & \\
\hline \multirow[t]{2}{*}{ Age (years) } & $2-12$ & 31 (27.2) & $11(9.6)$ & \multirow[t]{2}{*}{0.029} & \multirow[t]{2}{*}{0.173} \\
\hline & 13 and above & $41(41.4)$ & $6(6.1)$ & & \\
\hline \multirow[t]{4}{*}{ Main occupation } & Farmer & 27 (38.6) & $3(4.3)$ & \multirow[t]{4}{*}{0.567} & \multirow[t]{4}{*}{0.160} \\
\hline & At home & $15(27.3)$ & $8(14.5)$ & & \\
\hline & At school & 27 (33.3) & $6(7.4)$ & & \\
\hline & Other & $3(42.9)$ & $0(0.0)$ & & \\
\hline \multirow[t]{2}{*}{ Occupation } & Non-Agricultural & $39(28.5)$ & $14(10.1)$ & \multirow[t]{2}{*}{0.044} & \multirow[t]{2}{*}{0.114} \\
\hline & Agricultural & 32 (42.7) & $3(4.0)$ & & \\
\hline \multirow{2}{*}{$\begin{array}{l}\text { Type of indoor floor } \\
\text { covering in the } \\
\text { house }\end{array}$} & $\begin{array}{l}\text { Sanitary floor } \\
\text { (cement, wood, etc.) }\end{array}$ & $72(34.0)$ & $17(8.0)$ & \multirow[t]{2}{*}{0.474} & \multirow[t]{2}{*}{0.764} \\
\hline & Soil floor & $0(0.0)$ & $0(0.0)$ & & \\
\hline \multirow{2}{*}{$\begin{array}{l}\text { Type of yard } \\
\text { covering }\end{array}$} & Not soil & $72(34)$ & $3(8.5)$ & \multirow[t]{2}{*}{0.648} & \multirow[t]{2}{*}{0.888} \\
\hline & Soil & $0(0.0)$ & $14(7.9)$ & & \\
\hline \multirow[t]{2}{*}{$\begin{array}{l}\text { Waste water } \\
\text { treatment }\end{array}$} & $\begin{array}{l}\text { Sanitary waste water } \\
\text { treatment }\end{array}$ & $33(30.6)$ & $8(7.4)$ & \multirow[t]{2}{*}{0.310} & \multirow[t]{2}{*}{0.754} \\
\hline & $\begin{array}{l}\text { Without waste water } \\
\text { treatment }\end{array}$ & 39 (37.1) & $9(8.6)$ & & \\
\hline \multirow[t]{2}{*}{$\begin{array}{l}\text { Water sources for } \\
\text { daily activity }\end{array}$} & $\begin{array}{l}\text { Sanitary water } \\
\text { sources }\end{array}$ & 19 (20.7) & $8(8.7)$ & \multirow[t]{2}{*}{0.000} & \multirow[t]{2}{*}{0.737} \\
\hline & $\begin{array}{l}\text { Unsanitary water } \\
\text { sources }\end{array}$ & $53(43.8)$ & $9(7.4)$ & & \\
\hline \multirow[t]{2}{*}{ Drinking water } & $\begin{array}{l}\text { Sanitary drinking } \\
\text { water }\end{array}$ & 18 (15.4) & $13(11.1)$ & \multirow[t]{2}{*}{0.000} & \multirow[t]{2}{*}{0.63} \\
\hline & $\begin{array}{l}\text { Unsanitary drinking } \\
\text { water }\end{array}$ & $54(56.3)$ & $4(4.2)$ & & \\
\hline Toilet & $\begin{array}{l}\text { Sanitary toilet in the } \\
\text { home }\end{array}$ & 55 (30.4) & $14(7.7)$ & 0.012 & 0.752 \\
\hline
\end{tabular}




\section{Personal hygiene and prevalence of hookworm and S. stercoralis infections}

Hookworm infections were correlated with the use of shoes outdoors $(p=0.034)$, use of toilets at home $(p=0.048)$, and use of sandals at toilets $(p=0.004)$. S. stercoralis infections were not correlated significantly with personal hygiene. Data are shown in Table 4. 
Table 4

The correlation between personal hygiene and the prevalence of hookworm and S. stercoralis infections

\begin{tabular}{|c|c|c|c|c|c|}
\hline \multirow[t]{2}{*}{ Variable } & \multirow[t]{2}{*}{ Category } & \multicolumn{2}{|c|}{ Positive n (\%) } & \multicolumn{2}{|l|}{ P-value } \\
\hline & & Hookworm & $\begin{array}{l}S . \\
\text { stercoralis }\end{array}$ & Hookworm & $\begin{array}{l}\text { S. } \\
\text { stercoralis }\end{array}$ \\
\hline \multirow[t]{2}{*}{ Using shoes outdoors } & Routine & $32(43.2)$ & $4(5.4)$ & \multirow[t]{2}{*}{0.034} & \multirow[t]{2}{*}{0.311} \\
\hline & $\begin{array}{l}\text { Not } \\
\text { routine }\end{array}$ & $40(28.8)$ & $13(9.4)$ & & \\
\hline \multirow[t]{2}{*}{ Washing feet after soil contact } & Yes & $6(23.1)$ & $4(15.4)$ & \multirow[t]{2}{*}{0.217} & \multirow[t]{2}{*}{0.137} \\
\hline & No & $66(35.3)$ & $13(7.0)$ & & \\
\hline \multirow{2}{*}{$\begin{array}{l}\text { Washing fruits/vegetables } \\
\text { before eating }\end{array}$} & Routine & $8(24.2)$ & $2(6.1)$ & \multirow[t]{2}{*}{0.207} & \multirow[t]{2}{*}{0.658} \\
\hline & $\begin{array}{l}\text { Not } \\
\text { routine }\end{array}$ & $64(35.6)$ & $15(8.3)$ & & \\
\hline \multirow{2}{*}{$\begin{array}{l}\text { Eating raw/uncooked } \\
\text { fish/meat/vegetable }\end{array}$} & No & $63(36.6)$ & $11(6.4)$ & \multirow[t]{2}{*}{0.074} & \multirow[t]{2}{*}{0.080} \\
\hline & Yes & $9(22)$ & $6(14.6)$ & & \\
\hline \multirow[t]{2}{*}{ Pet contact } & No & $60(37.3)$ & $13(8.1)$ & \multirow[t]{2}{*}{0.060} & \multirow[t]{2}{*}{0.930} \\
\hline & Yes & $12(23.1)$ & $4(7.3)$ & & \\
\hline \multirow[t]{2}{*}{ Washing hands after pet contact } & Yes & $0(0.0)$ & $0(0.0)$ & \multirow[t]{2}{*}{0.149} & \multirow[t]{2}{*}{0.551} \\
\hline & No & $72(34.4)$ & $17(8.1)$ & & \\
\hline \multirow{2}{*}{$\begin{array}{l}\text { Washing hands after soil } \\
\text { contact }\end{array}$} & Yes & $6(24.0)$ & $4(16.0)$ & \multirow[t]{2}{*}{0.270} & \multirow[t]{2}{*}{0.115} \\
\hline & No & $66(35.1)$ & $13(6.9)$ & & \\
\hline \multirow{2}{*}{$\begin{array}{l}\text { Washing feet before entering } \\
\text { house }\end{array}$} & No & $7(53.8)$ & $0(0.0)$ & \multirow[t]{2}{*}{0.115} & \multirow[t]{2}{*}{0.273} \\
\hline & Yes & $65(32.5)$ & $17(8.5)$ & & \\
\hline \multirow[t]{2}{*}{ Using the toilet at home } & Yes & $46(39.7)$ & $10(6.8)$ & \multirow[t]{2}{*}{0.048} & \multirow[t]{2}{*}{0.706} \\
\hline & No & $26(26.8)$ & $7(7.2)$ & & \\
\hline \multirow[t]{2}{*}{ Using sandals at the toilet } & Routine & $46(39.7)$ & $10(8.6)$ & \multirow[t]{2}{*}{0.048} & \multirow[t]{2}{*}{0.706} \\
\hline & $\begin{array}{l}\text { Not } \\
\text { routine }\end{array}$ & $26(26.8)$ & $7(7.2)$ & & \\
\hline
\end{tabular}

\section{Environmental risk factors of hookworm and S. stercoralis infections}

The results of the chi-square analysis on the environmental risk factors in regard to the high prevalence of hookworm infection and low prevalence of S. stercoralis infection in East Kalimantan Province are 
shown in Table 5 below.

Page 14/23 
Table 5

Environmental risk factors of hookworm and S. stercoralis infections in East Kalimantan Province

\begin{tabular}{|c|c|c|c|c|c|}
\hline \multirow[t]{2}{*}{ Variable } & \multirow[t]{2}{*}{ Category } & \multicolumn{2}{|c|}{ Positive n (\%) } & \multicolumn{2}{|l|}{$P$-value } \\
\hline & & Hookworm & $\begin{array}{l}\text { S. } \\
\text { stercoralis }\end{array}$ & Hookworm & $\begin{array}{l}\text { S. } \\
\text { stercoralis }\end{array}$ \\
\hline \multirow[t]{2}{*}{ District } & Muara Kaman & $53(55.8)$ & $3(3.2)$ & \multirow[t]{2}{*}{0.000} & \multirow[t]{2}{*}{0.020} \\
\hline & Marangkayu & $19(16.1)$ & $14(11.9)$ & & \\
\hline \multirow{2}{*}{$\begin{array}{l}\text { Organic carbon content } \\
\text { in soil (\%) }\end{array}$} & $1.37-2.47$ & $21(23.1)$ & $13(14.3)$ & \multirow[t]{2}{*}{0.004} & \multirow[t]{2}{*}{0.003} \\
\hline & $>2.47-4.04$ & $51(41.8)$ & $4(3.3)$ & & \\
\hline \multirow[t]{2}{*}{$\mathrm{pH}$ of soil } & $4.26-5.85$ & $31(30.4)$ & $7(6.9)$ & \multirow[t]{2}{*}{0.313} & \multirow[t]{2}{*}{0.564} \\
\hline & $>5.85-6.92$ & $41(36.9)$ & $10(9.0)$ & & \\
\hline \multirow[t]{2}{*}{ Clay content in soil (\%) } & $4-18.5$ & $35(36.5)$ & $7(7.3)$ & \multirow[t]{2}{*}{0.458} & \multirow[t]{2}{*}{0.737} \\
\hline & $>18.5-42.50$ & $37(31.6)$ & $10(8.5)$ & & \\
\hline \multirow[t]{2}{*}{ Temperature $\left({ }^{0} \mathrm{C}\right)$} & $28-28.6$ & $19(16.1)$ & $3(3.2)$ & \multirow[t]{2}{*}{0.000} & \multirow[t]{2}{*}{0.020} \\
\hline & $>28.6-29.5$ & $53(55.8)$ & $14(11.9)$ & & \\
\hline \multirow[t]{2}{*}{ Humidity (\%) } & 65 & $19(16.1)$ & $14(11.9)$ & \multirow[t]{2}{*}{0.000} & \multirow[t]{2}{*}{0.020} \\
\hline & 66 & $53(55.8)$ & $3(3.2)$ & & \\
\hline \multirow{2}{*}{$\begin{array}{l}\text { Number of days of } \\
\text { rainfall (day) }\end{array}$} & 164 & $53(55.8)$ & $3(3.2)$ & \multirow[t]{2}{*}{0.000} & \multirow[t]{2}{*}{0.020} \\
\hline & 174 & $19(16.1)$ & $14(11.9)$ & & \\
\hline \multirow[t]{2}{*}{ Rainfall volume $\left(\mathrm{mm}^{3}\right)$} & 3,549 & $53(55.8)$ & $3(3.2)$ & \multirow[t]{2}{*}{0.000} & \multirow[t]{2}{*}{0.020} \\
\hline & 4,000 & $19(16.1)$ & $14(11.9)$ & & \\
\hline \multirow{2}{*}{$\begin{array}{l}\text { Elevation above sea } \\
\text { level }(\mathrm{m})\end{array}$} & $15-41.6$ & $53(55.8)$ & $3(3.1)$ & \multirow[t]{2}{*}{0.000} & \multirow[t]{2}{*}{0.016} \\
\hline & $>41.6-50$ & $19(16.1)$ & $14(12.1)$ & & \\
\hline \multirow[t]{2}{*}{ Texture of soil } & $\begin{array}{l}\text { Sandy soil with } \\
\text { organic material }\end{array}$ & $46(33.1)$ & $14(10.1)$ & \multirow[t]{2}{*}{0.764} & \multirow[t]{2}{*}{0.123} \\
\hline & $\begin{array}{l}\text { Non-sandy soil } \\
\text { with organic } \\
\text { material }\end{array}$ & $26(35.1)$ & $3(4.1)$ & & \\
\hline Vegetation & $\begin{array}{l}\text { Palm plantations } \\
\text { and/or rubber } \\
\text { plantations }\end{array}$ & 65 (38.9) & $12(7.2)$ & 0.003 & 0.414 \\
\hline & Rice fields & $7(15.2)$ & $5(10.9)$ & & \\
\hline Village areas & Buffer river/sea & 65 (39.2) & $12(7.2)$ & 0.002 & 0.446 \\
\hline
\end{tabular}




\begin{tabular}{|c|c|c|c|c|c|}
\hline & Hill area & $7(14.9)$ & $5(10.6)$ & & \\
\hline \multirow{2}{*}{$\begin{array}{l}\text { Dry or wet soil } \\
\text { surrounding the house }\end{array}$} & Dry soil & $51(30.2)$ & $15(8.9)$ & \multirow[t]{2}{*}{0.028} & \multirow[t]{2}{*}{0.345} \\
\hline & Wet soil & $21(47.7)$ & $2(4.5)$ & & \\
\hline \multirow[t]{2}{*}{ Having cats as pets } & No & $30(37.5)$ & $5(6.3)$ & \multirow[t]{2}{*}{0.376} & \multirow[t]{2}{*}{0.470} \\
\hline & Yes & $42(31.6)$ & $12(9.0)$ & & \\
\hline \multirow[t]{2}{*}{ Having dogs as pets } & No & $0(0.0)$ & $0(0.0)$ & \multirow[t]{2}{*}{0.149} & \multirow[t]{2}{*}{0.552} \\
\hline & Yes & $72(34.4)$ & $17(8.1)$ & & \\
\hline \multirow{2}{*}{$\begin{array}{l}\text { Hookworm/S. } \\
\text { stercoralis in cats }\end{array}$} & Negative & $0(0.0)$ & $10(7.5)$ & \multirow[t]{2}{*}{0.474} & \multirow[t]{2}{*}{0.716} \\
\hline & Positive & $72(34.4)$ & $7(8.9)$ & & \\
\hline \multirow{2}{*}{$\begin{array}{l}\text { Hookworm/S. } \\
\text { stercoralis in dogs }\end{array}$} & Negative & $0(0.0)$ & $1(1.9)$ & \multirow[t]{2}{*}{0.000} & \multirow[t]{2}{*}{0.064} \\
\hline & Positive & $72(34.4)$ & $9(9.9)$ & & \\
\hline \multirow{2}{*}{$\begin{array}{l}\text { Habitation with humans } \\
\text { infected with hookworm } \\
\text { and/or } S \text {. stercoralis }\end{array}$} & No & $43(26.3)$ & $2(1.0)$ & \multirow[t]{2}{*}{0.007} & \multirow[t]{2}{*}{0.000} \\
\hline & Yes & $29(47.5)$ & 15 (78.9) & & \\
\hline
\end{tabular}

Hookworm infection was significantly correlated with the locality of the district $(p=0.000)$, organic carbon content in the soil $(p=0.004)$, temperature $(p=0.000)$, humidity $(p=0.000)$, number of days of rainfall $(p=0.000)$, rainfall volume $(p=0.000)$, elevation above sea level $(p=0.000)$, surrounding vegetation $(p=0.003)$, village areas $(p=0.002)$, dry or wet soil surrounding houses $(p=0.028)$, hookworm in dogs $(p=0.000)$, and habitation with humans infected with hookworm $(p=0.001)$. S. stercoralis infection was significantly correlated with the locality of the district $(p=0.020)$, organic carbon content in the soil $(p=0.003)$, temperature $(p=0.020)$, humidity $(p=0.020)$, number of days of rainfall $(p=0.020)$, rainfall volume $(p=0.020)$, and elevation above sea level $(p=0.016)$.

\section{Discussion}

The prevalence of hookworm infections showed higher than in S. stercoralis infections in rural East Kalimantan, Indonesia. In this study, we found that the prevalence of hookworm infection was higher in Muara Kaman District than in Marangkayu District, while the prevalence S. stercoralis infection was higher in Marangkayu District than in Muara Kaman District. The difference in environmental factors between Muara Kaman District and Marangkayu District was that Muara Kaman District is surrounded by palm plantations and river area, which might affect the increasing or decreasing prevalence of $S$. stercoralis infection in rural areas (2). A similar study was performed in Manufahi District, Timor Leste, with a prevalence of hookworm infection at $62.8 \%$ (17). 
In regard to the organic carbon content in the soil, hookworm infection was higher in the category $>2.47-$ $4.04 \%$ than in the category $1.37-2.47 \%$ with prevalence at 51 (41.8\%) and $21(23.1 \%)$, respectively. $S$. stercoralis infection was higher in the category $1.37-2.47$ than in the category $>2.47-4.04 \%$ with prevalence at $13(14.3 \%)$ and 4 (3.3\%), respectively. The organic carbon content in the soil with the category $>2.47-4.04 \%$ was 1.70 times more likely for hookworm infection than in the category $1.37-$ $2.47 \%$, while the organic carbon content in the soil with the category $1.37-2.47 \%$ was 2.56 times more likely for $S$. stercoralis infection than in the category $>2.47-4.04 \%$. A Cambodian study explained that high amounts of organic carbon content in the soil might not affect the prevalence of hookworm infection but might affect the prevalence of $S$. stercoralis infection (11). This may indicate that the organic carbon content in the soil in East Kalimantan Province might not reduce hookworm infection but might reduce $S$. stercoralis infection. From the study of Sebastian $\mathrm{H}$, et al., the organic carbon content in the soil had an indirect effect on nematodes, but the effect of the organic carbon content via food organisms was usually on the abundance, cell size, and activity of bacteria (Escherichia coli) (18). Absorption of organic carbon by bacterial cells, serving as food for the nematodes, may be an important factor for the availability of organic carbon for hookworm.

The present study demonstrated that hookworm infection was associated with temperature, humidity, number of days of rainfall, rainfall volume, and elevation above sea level. These results were supported by a previous study that demonstrated that the rainy season may reduce the prevalence of hookworm and S. stercoralis infections (10). Another previous study also supported the results of this study by demonstrating that elevation contributed to the distribution of the prevalence hookworm and $S$. stercoralis infections (19). Environmental factors in East Kalimantan Province might be similar to Southern Thailand including long rainy seasons, temperature, and several geographical characteristics, which might have an impact on the higher prevalence of hookworm infection in East Kalimantan Province than in Southern Thailand (10). Another study in Southern Laos demonstrated a high prevalence of $S$. stercoralis infection during heavy rainfall with poor sanitation (12).

Hookworm infection was higher in households surrounded by vegetation and villages surrounded by palm plantations and/or rubber plantations than in villages surrounded by rice fields with the prevalence at 65 (38.9\%) and 7 (15.2\%), respectively; while S. stercoralis infection was higher in villages surrounded by rice fields than those surrounded by palm plantations and/or rubber plantations with the prevalence at $5(10.9 \%)$ and $12(7.2 \%)$, respectively. The reason is because the surrounding palm plantations have a higher temperature than the rice fields, which provide a suitable environment for hookworm but not for $S$. stercoralis. In addition, the soil covering at palm plantations might be a potential source for hookworm transmission rather than $S$. stercoralis infection, whereas rice fields might be a potential source for $S$. stercoralis infection during farming activity (20).

The prevalence of hookworm infection was higher in village areas with a river or coastal areas than in hill areas, while the prevalence of $S$. stercoralis infection was higher in hill areas. In addition, hookworm infection was more likely in houses surrounded by wet soil rather than dry soil, while $S$. stercoralis infection was more likely in houses surrounded by dry soil rather than wet soil. Hookworm and $S$. 
stercoralis infections were more likely in participants who have dogs than those who do not have dogs. Dogs were a potential risk of transmitting hookworm and S. stercoralis among communities in rural villages with poor sanitation and a low quality of personal hygiene among members of the household.

The results of this study were supported by a similar research conducted among Cambodian participants, where dogs were a serious risk factor for hookworm and S. stercoralis infections. Moreover, a previous study also demonstrated that dogs have a higher potential risk for hookworm and $S$. stercoralis infection than cats $(21,22)$. A study in Southern Thailand also demonstrated that dogs were correlated with hookworm and S. stercoralis infections (23). The factors related to the risk of hookworm and $S$. stercoralis infections consisted of poor hygiene, including infrequent hand washing and unclean feet after soil contact. These factors supported the penetration of $S$. stercoralis larvae to the human host (23, 24).

\section{Conclusions}

The prevalence of hookworm infection was higher than S. stercoralis infection in rural areas of East Kalimantan Province. The higher prevalence of hookworm infection than $S$. stercoralis infection might be due to environmental factors, sanitation conditions, and personal hygiene of the participants.

Environmental factors such as the quality of the soil, length of rainy season, surrounding vegetation, and village areas were significant factors for increasing hookworm infection. In contrast, some of these factors became preventative factors for the prevalence of $S$. stercoralis infection. Demographic characteristics, sanitation conditions, and personal hygiene that were correlated with hookworm and $S$. stercoralis infections should be used for the control or reduction of hookworm and S. stercoralis infections in the rural areas of East Kalimantan Province.

\section{List Of Abbreviations}

S. stercoralis; Strongyloides stercoralis, OR; odds ratio, STH; soil-transmitted helminthes.

\section{Declarations}

\section{Ethical approval and consent to participate}

The study protocol was approved by the Ethical Clearance Committee on human rights related to research involving human subjects, Walailak University (HE: No. WUEc-18-034-01).

\section{Consent for publication}

Official permission and ethical clearance for collection of human fecal samples were obtained from the head of the village and the head of the household.

\section{Availability of supporting data}


All data related to the present study in this manuscript are available.

\section{Competing interests}

The authors declare that there is no conflict of interest regarding the publication of this article.

\section{Funding}

This work was supported by a grant from Walailak University (Contract No. 17/2561) and a grant for PhD studies from the East Kalimantan Provincial Government, Indonesia.

\section{Authors' contributions}

All authors conceived of the idea and participated in the design of this study. BS, JT, and MK conducted the study. BS, SW, SP, WA, and JT were responsible for the interpretation of the results, drafting of the manuscript, and reading for intellectual content. All authors read and approved the final manuscript.

\section{Acknowledgments}

We are grateful to the participants and local authorities of Muara Kaman District and Marangkayu District, East Kalimantan Province, Indonesia. We would like to deeply thank the Dean of the School of Allied Health, Walailak University, and the Dean of the School of Public Health, Mulawarman University, for permitting us to use their laboratory and for supporting us through an approval letter for this research.

\section{References}

1. Wardell R, Clements, A. C., Lal, A., Summers, D., Llewellyn, S., Campbell, S. J., ... \& Nery, S. V. An environmental assessment and risk map of Ascaris lumbricoides and Necator americanus distributions in Manufahi District, Timor-Leste. PLoS neglected tropical diseases. 2017;11(5):e0005565.

2. Pullan RL, Smith JL, Jasrasaria R, Brooker SJ. Global numbers of infection and disease burden of soil transmitted helminth infections in 2010. Parasites \& vectors. 2014;7(1):37.

3. Organization WH. Helminth control in school-age children: a guide for managers of control programmes: World Health Organization; 2011.

4. Bethony J, Brooker S, Albonico M, Geiger SM, Loukas A, Diemert D, et al. Soil-transmitted helminth infections: ascariasis, trichuriasis, and hookworm. The lancet. 2006;367(9521):1521-32.

5. Forrer A, Khieu V, Schär F, Vounatsou P, Chammartin F, Marti H, et al. Strongyloides stercoralis and hookworm co-infection: spatial distribution and determinants in Preah Vihear Province, Cambodia. Parasites \& vectors. 2018;11(1):33.

6. Bannon JP, Fater M, Solit R. Intestinal ileus secondary to Strongyloides stercoralis infection: case report and review of the literature. The American surgeon. 1995;61(4):377. 
7. Hall A, Conway DJ, Anwar KS, Rahman ML. Strongyloides stercoralis in an urban slum community in Bangladesh: factors independently associated with infection. Transactions of the Royal Society of Tropical Medicine and Hygiene. 1994;88(5):527-30.

8. Anamnart W, Pattanawongsa A, Intapan PM, Morakote N, Janwan P, Maleewong W. Detrimental effect of water submersion of stools on development of Strongyloides stercoralis. PLoS One. 2013;8(12):e82339.

9. Na-Ek P, Sanpool O, Jongthawin J, Anamnart W, Intapan PM, Chamavit P, et al. Restoration of hookworm egg development after prolonged storage in stool suspension. Parasitology research. 2016;115(7):2817-23.

10. Anamnart W, Intapan PM, Pattanawongsa A, Chamavit P, Kaewsawat S, Maleewong W. Effect of dilution of stool soluble component on growth and development of Strongyloides stercoralis. Scientific Reports. 2015;5(1):1-5.

11. Khieu V, Schär F, Forrer A, Hattendorf J, Marti H, Duong S, et al. High prevalence and spatial distribution of Strongyloides stercoralis in rural Cambodia. PLoS Negl Trop Dis. 2014;8(6):e2854.

12. Vonghachack Y, Sayasone S, Bouakhasith D, Taisayavong K, Akkavong K, Odermatt P. Epidemiology of Strongyloides stercoralis on Mekong islands in southern Laos. Acta tropica. 2015;141:289-94.

13. Daniel WW. A foundation for analysis in the health sciences. Biostatistics New York: Wiley. 1991.

14. Koga K, Kasuya S, Khamboonruang C, Sukhavat K, leda M, Takatsuka N, et al. A modified agar plate method for detection of Strongyloides stercoralis. The American journal of tropical medicine and hygiene. 1991;45(4):518-21.

15. Katz N, Chaves A, Pellegrino J. A simple device for quantitative stool thick-smear technique in schistosomiasis mansoni. Rev Inst Med Trop Sao Paulo. 1972;14(6):397-400.

16. Anamnart W, Pattanawongsa A, Intapan PM, Maleewong W. Albendazole stimulates the excretion of Strongyloides stercoralis larvae in stool specimens and enhances sensitivity for diagnosis of strongyloidiasis. Journal of clinical microbiology. 2010;48(11):4216-20.

17. Nery SV MJ, Traub R, et al. A cluster-randomized controlled trial intregrating a community-based water, sanitation and hygiene program, with mass distribution of albendazole to reduce intestinal parasites in Timor-Leste: the WASH for WORMS research protocol. BMJ Open 2015;5:e009293.

18. Höss S, Bergtold M, Haitzer M, Traunspurger W, Steinberg CE. Refractory dissolved organic matter can influence the reproduction of Caenorhabditis elegans (Nematoda). Freshwater Biology. 2001;46(1):1-10.

19. Raso G, Vounatsou P, Gosoniu L, Tanner M, N'Goran EK, Utzinger J. Risk factors and spatial patterns of hookworm infection among schoolchildren in a rural area of western Côte d'Ivoire. International journal for parasitology. 2006;36(2):201-10.

20. Alcaraz CO, Adell RI, Sánchez PS, Blasco MJV, Sánchez OA, Auñón AS, et al. Characteristics and geographical profile of strongyloidiasis in healthcare area 11 of the Valencian community (Spain). Journal of infection. 2004;49(2):152-8. 
21. Schär F, Inpankaew T, Traub RJ, Khieu V, Dalsgaard A, Chimnoi W, et al. The prevalence and diversity of intestinal parasitic infections in humans and domestic animals in a rural Cambodian village. Parasitology international. 2014;63(4):597-603.

22. Štrkolcová G, Goldova M, Bockova E, Mojžišová J. The roundworm Strongyloides stercoralis in children, dogs, and soil inside and outside a segregated settlement in Eastern Slovakia: frequent but hardly detectable parasite. Parasitology research. 2017;116(3):891-900.

23. Punsawad C, Phasuk N, Bunratsami S, Thongtup K, Siripakonuaong N, Nongnaul S. Prevalence of intestinal parasitic infection and associated risk factors among village health volunteers in rural communities of southern Thailand. BMC public health. 2017;17(1):1-9.

24. Garcia LS. Diagnostic medical parasitology: American Society for Microbiology Press; 2006.

\section{Figures}

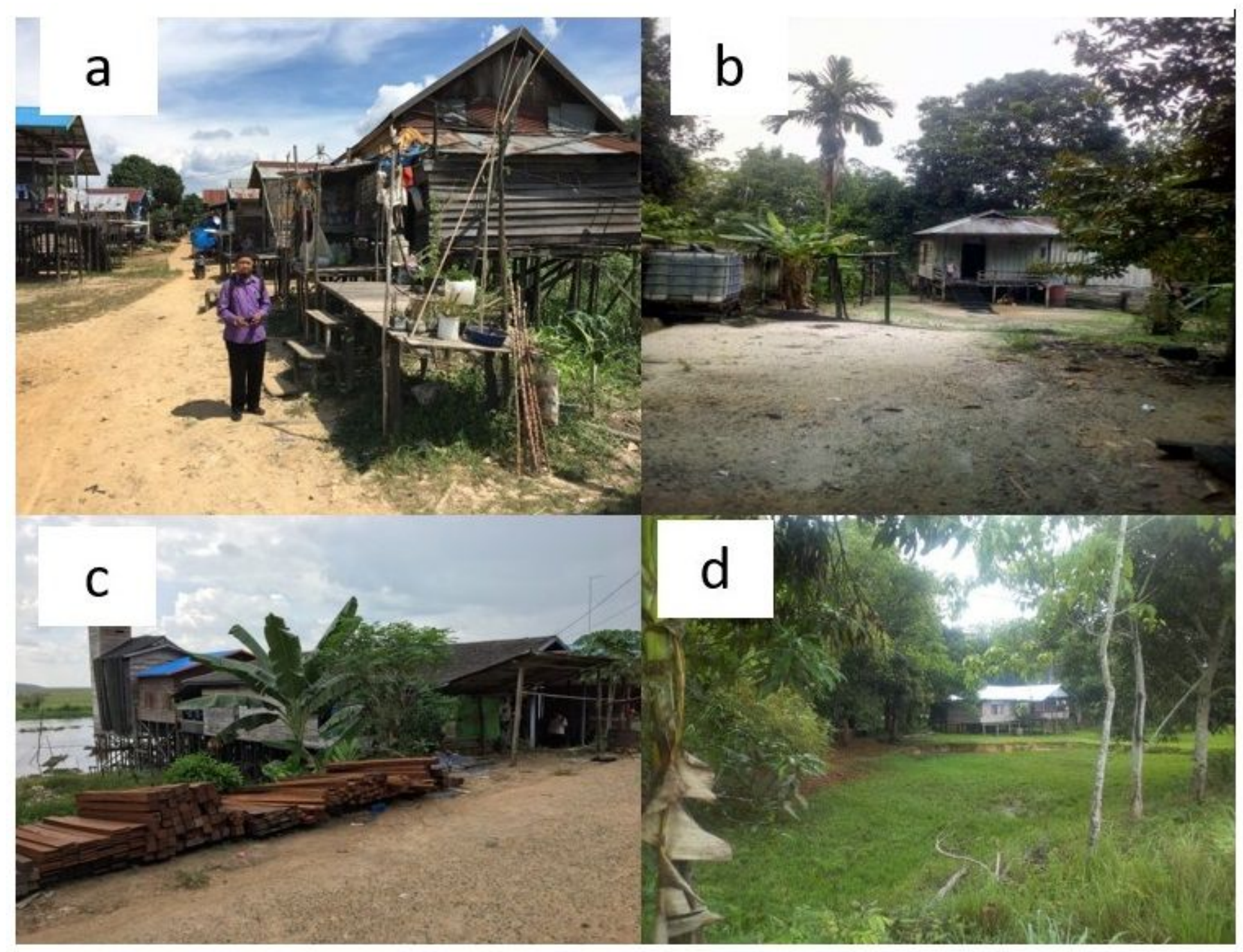

\section{Figure 1}


Data collection (a, b, c, d) in rural areas of Muara Kaman District and Marangkayu District, East Kalimantan Province

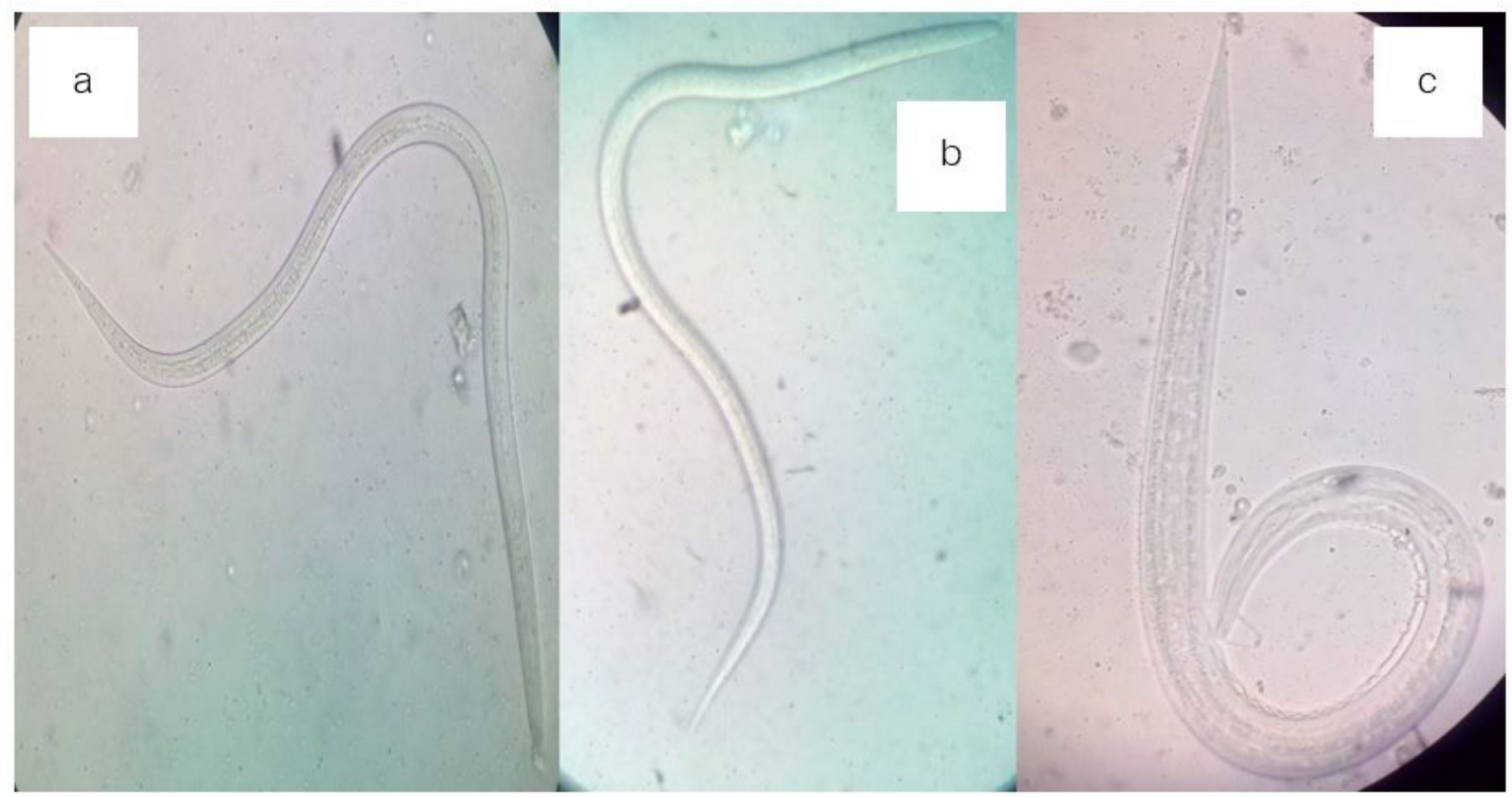

\section{Figure 2}

Modified agar plate techniques. (a, b, c) Hook worm filariform larva (L3). Microscopic observation under low power $(x 10)$

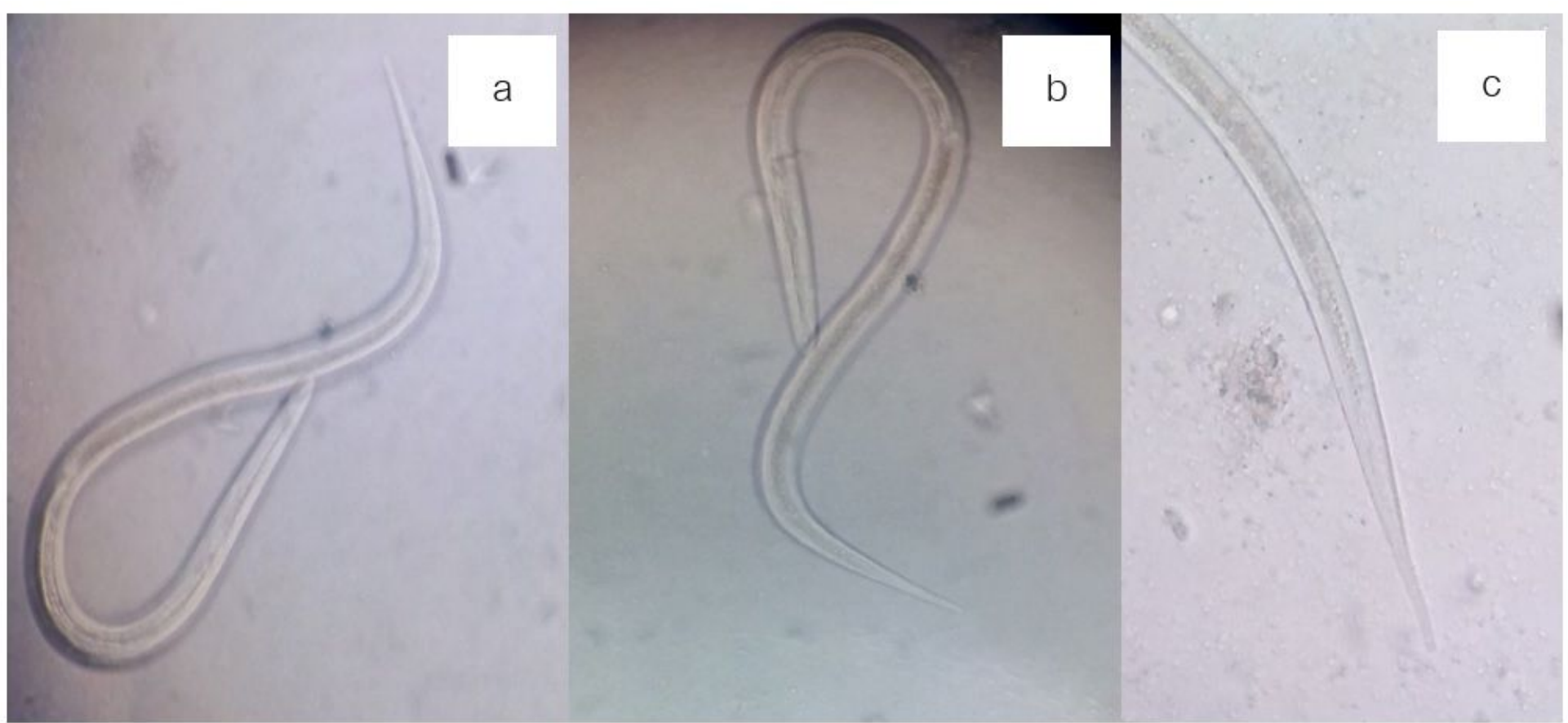

Figure 3 
Modified agar plate techniques. $(a, b, c)$ Strongyloides stercoralis filariform larva (L3). Microscopic observation under low power (x10). 